\title{
Modelagem matemática da curva de carga de um sistema elétrico real a partir de séries de Fourier
}

\author{
Andressa Tais Diefenthäler ${ }^{1}$, Airam T. Z. R. Sausen ${ }^{1}$, Paulo S. Sausen ${ }^{1}$ and \\ Maurício de Campos ${ }^{1}$ \\ ${ }^{1}$ Universidade Regional do Noroeste do Estado do Rio Grande do Sul (UNIJUI) \\ *andressa_td@hotmail.com; airamsausen@gmail.com; paulosausen@gmail.com; decampos.mauricio@gmail.com
}

Recebido: 29/10/2018. Revisado: 17/06/2019. Aceito: 25/06/2019.

\begin{abstract}
Resumo
O conhecimento da curva típica de carga é fundamental para o planejamento e a operação de redes de distribuição de energia elétrica, principalmente diante das tendências da geração distribuída e de transformação dos sistemas em Smart Grids. Assim sendo, este artigo apresenta a modelagem matemática, a partir de séries de Fourier, da curva de carga diária de dois alimentadores de uma rede real de distribuição de energia elétrica do sul do Brasil. Com a utilização do software Matlab (student license), considerando o método dos mínimos quadrados não linear e dados reais referentes a carga consumida nos anos de 2016 a 2018, foram ajustadas curvas do tipo série de Fourier para cada estação climática e alimentador, obtendo-se modelos matemáticos com elevada acurácia. A análise dos resultados possibilita o conhecimento da demanda e do perfil da carga, identificando os períodos de pico de consumo, assim como as variações horárias e sazonais. Através dessa pesquisa foram validados dois modelos matemáticos que permitem representar a curva típica de carga de um sistema e caracterizá-lo quanto ao tipo de carga predominante, sendo ela industrial ou residencial.
\end{abstract}

Palavras-Chave: Curva típica de carga; industrial e residencial; modelagem matemática; série de Fourier.

\begin{abstract}
The knowledge of the typical load curve is fundamental for the planning and operation of electric distribution networks, especially in face of the trends of distributed generation and transformation of systems in Smart Grids. Thus, this paper presents the mathematical modeling, from the Fourier series, of the daily load curve of two feeders of a real electric distribution network of the Brazil south. Matlab software (student license) is used considering the nonlinear least squares method and real data of the load consumed in the years 2016 to 2018, Fourier series curves were adjusted for each climatic season and feeder, obtaining mathematical models with high accuracy. The analysis of the results allows the demand knowledge and the load profile identifying the peak consumption periods, as well as the hourly and seasonal variations. Through this research two mathematical models were validated. They allow to represent the typical load curve of a system and characterize it as to the type of predominant load, whether industrial or residential.
\end{abstract}

Key words: Fourier Series; industrial and residential; mathematical modeling; typical load curve.

\section{Introdução}

O Sistema Elétrico de Potência (SEP) brasileiro foi desenvolvido no século $\mathrm{XX}$, e nos últimos anos, vêm sofrendo inúmeras modificações, principalmente devido a crescente comercialização de equipamentos elétricos e eletrônicos, que tem ampliado a demanda dos consumidores, alterando seu perfil de consumo. Dentre as alterações nos SEPs, destacase a incorporação de tecnologias de informação e automação na rede elétrica, as quais estão 
relacionadas ao conceito de Smart Grids (i.e., Redes Inteligentes) (Macedo et al.; 2015). Essa nova geração de sistemas elétricos visa a modernização do SEP, tornando-o mais seguro, eficiente e econômico, atendendo à crescente demanda pelo fornecimento de energia elétrica de qualidade (Deng et al.; 2015).

Essas alterações nas redes ainda permitirão o fluxo de energia bidirecional entre o sistema e o consumidor, englobando, portanto, a Geração Distribuída (GD), a qual pode ser entendida como a geração de energia elétrica que é realizada na rede de distribuição ou junto ao consumidor, podendo ser de diferentes potências e envolver diversas fontes e tecnologias (Gampa and Das; 2015) (Ackermann et al.; 2001). A GD envolve, além da fonte de geração de energia (geralmente renovável), equipamentos de medição e controle, previstos pelas Smart Grids, que articulam a operação dos geradores e o controle de cargas para se adaptar a oferta de energia disponível, possibilitando obter maior eficiência energética (Xue et al.; 2008).

Nesse contexto, destaca-se a importância do conhecimento prévio da demanda dos consumidores, a qual pode ser definida como a carga nos terminais receptores, tomada em valor médio durante um determinado intervalo de tempo (Jiménez et al.; 2017) (Balachandra and Chandru; 1999). Essas cargas são classificadas em residencial, comercial, industrial, rural, iluminação pública e serviços públicos. Cada um desses segmentos apresenta um comportamento de consumo diferente e também, tarifações distintas (ANEEL; 2018). Além disso, a carga sofre variações devido à localização geográfica, os aspectos socioeconômicos, os horários do dia e dias da semana, a presença de feriados, e as variações sazonais, visto que alterações no clima influenciam diretamente no comportamento dos consumidores (González-Cabrera et al.; 2018) (Marques et al.; 2016).

A análise do perfil de consumo é comumente realizada a partir da construção de curvas típicas de carga, que podem ser entendidas como o registro horário, em um período diário, das demandas de capacidade para os diferentes tipos de consumidores (ANEEL; 2018). O conhecimento dessa curva é de grande importância para as concessionárias e, a partir do desenvolvimento das Smart Grids, também será fundamental para os consumidores, pois permite detectar os pontos de demanda máxima e mínima, favorecendo mudanças de hábitos de consumo e o planejamento dos sistemas (Macedo et al.; 2015). Por outro lado, as variações temporais e sazonais afetam diretamente as curvas de carga, representando um desafio à operação do SEP (Balachandra and Chandru; 1999) (Marques et al.; 2016).

Atualmente, diferentes pesquisas da literatura técnica (Souza and et al.; 2010) (Rahde and Kaehler; 2000) (Grandjean et al.; 2012) apresentam a obtenção da curva de carga considerando cenários específicos, tais como: consumidores individuais, classes de consumo, ou mesmo tipo de consumidor. Entretanto, não são encontrados trabalhos que analisam a curva de carga média de um alimentador da rede elétrica de distribuição, levando em consideração todos os consumidores deste segmento, e a carga real de consumo, por um período significativo de tempo (abrangendo as diferentes estações climáticas do ano). O estudo da curva de carga média geral de um alimentador é fundamental, visto que disparidades na demanda de potência entre os alimentadores, assim como a diversidade das cargas instaladas, podem ocasionar inúmeros impactos, como a sobrecarga de alimentadores em horários diferentes ao longo do dia e o consequente aumento dos níveis de perdas técnicas, resultando na redução da confiabilidade do sistema, em conjunto com significativas perdas econômicas para a concessionária (Santos et al.; 2017).

Diferentemente deste trabalho, em que é desenvolvido um modelo matemático, os trabalhos correlatos ao tema (Taylor; 2003) (Amjady; 2001) (Magnano and Boland; 2007) utilizam diferentes técnicas de previsão de demanda de carga, a partir de algoritmos de descoberta de conhecimento/mineração de dados, redes neurais artificiais, ou ainda modelos do tipo ARMA (autoregressive moving average). Existem ainda trabalhos (González-Cabrera et al.; 2018) (Zhang et al.; 2008) (Oliveira et al.; 2006) que realizam a representação da curva de carga de um sistema a partir da representação gráfica dos dados da demanda, ou mesmo através da interpolação destes pontos, sem a preocupação de disponibilizar um modelo matemático que permita representar, simular e caracterizar a curva de carga gerada.

Neste contexto, no presente artigo é apresentado o desenvolvimento da modelagem matemática da curva de carga de dois alimentadores de uma concessionária de energia elétrica do sul do Brasil, utilizando séries de Fourier. A partir do estudo, são disponibilizados dois modelos matemáticos validados que possibilitam descrever e caracterizar a curva típica de carga de um sistema elétrico, de acordo com o perfil de consumo industrial ou residencial predominante no sistema.

Os modelos matemáticos são obtidos e validados a partir de dados reais fornecidos pelo Departamento Municipal de Energia Elétrica da cidade de Ijuí (DEMEI), que é uma concessionária de energia da região noroeste do estado do Rio Grande do Sul (RS). Os dados são referentes a carga consumida em quatro alimentadores da rede entre os anos de 2016 a 2018. Como ferramenta computacional é utilizado o software Matlab (student license), no qual é utilizado o método dos mínimos quadrados não linear. Os resultados das simulações são analisados a partir de representações gráficas e do cálculo do coeficiente de determinação.

O restante desse artigo está organizado como segue. Na Seção 2 é apresentada uma pequena introdução sobre o conceito das Smart Grids e a geração distribuída. Na Seção 3 são abordados trabalhos relacionados à curva típica de carga e o perfil de consumo brasileiro. A metodologia adotada nessa pesquisa é descrita na Seção 4. Na Seção 5 são expostos os resultados e discussão. Por fim, as conclusões e possibilidades de trabalhos futuros são apresentados na Seção 6.

\section{Smart grids e a geração distribuída}

As Smart Grids envolvem a utilização de tecnologias da comunicação, automação e computação na rede elétrica, provocando profundas modificações, principalmente nos sistemas de informação, medição e proteção do SEP (Macedo et al.; 2015). A partir da incorporação de novos recursos e equipamentos, 
é possível desenvolver um sistema elétrico mais eficiente, confiável, flexível, otimizado, econômico e ambientalmente correto (Deng et al.; 2015).

As Smart Grids preveem o fluxo de energia e de informação bidirecional entre o sistema de fornecimento e o consumidor, considerando a possibilidade de armazenamento de energia e a utilização de sistemas automáticos de medição (Macedo et al.; 2015). Assim, ela viabiliza a GD, a qual apresenta vantagens em relação à geração centralizada, pois, de modo geral, causa baixos impactos ambientais, e auxilia na economia de investimentos na transmissão e distribuição de energia. A alocação ótima da GD também auxilia no atendimento da demanda nos momentos de pico de consumo, e prevê a divulgação de informações, tais como acerca das tarifas diferenciadas ao longo do dia, o que favorece o planejamento dos consumidores quanto a diminuir o consumo ou deslocar o pico da demanda para períodos com cargas mais leves, sem sobrecarregar o sistema, viabilizando o Gerenciamento pelo Lado da Demanda (GLD) (Souza and et al.; 2010) (Rahde and Kaehler; 2000).

Para a efetivação de todos estes benefícios, tanto para a concessionária como para o consumidor, é de fundamental importância o conhecimento da curva típica de carga do sistema (Gampa and Das; 2015) (Ackermann et al.; 2001). Esse conhecimento também é relevante diante do aumento crescente do consumo de equipamentos elétricos e eletrônicos, que tem ocorrido nos últimos anos. Adiciona-se a este consumo, especialmente no Brasil, o início da comercialização de veículos elétricos, que pode provocar significativo acréscimo na demanda e, consequentemente, modificações na curva de carga, até então típica do sistema elétrico (Deng et al.; 2015).

\section{Curvas típicas de carga}

Diante da importância de conhecer a demanda e o comportamento das cargas consumidoras de energia elétrica, diferentes pesquisadores, ao longo das últimas décadas, têm modelado o perfil de consumo, visando obter uma ferramenta que permita prever, quantificar e planejar a geração e o fornecimento de energia, além do controle e gerenciamento dos sistemas elétricos, de acordo com a região e o tipo de consumidor (Deng et al.; 2015) (Grandjean et al.; 2012).

Dentre os trabalhos da literatura, destacam-se os que se dedicam a obtenção de modelos de previsão de demanda, a curto ou longo prazo, considerando diferentes variáveis e métodos. Em Jiménez et al. (2017) é proposta uma metodologia para a determinação das variáveis de entrada e dos parâmetros de modelos de predição de demanda de energia elétrica, baseados em redes neurais artificiais, considerando o impacto de fatores como o dia da semana, o horário do dia e o clima em uma cidade do Caribe.

As pesquisas desenvolvidas em Taylor (2003) e Amjady (2001), propõem a modelagem de séries temporais de previsão de carga de curto prazo, considerando modelos ARMA. Em Magnano and Boland (2007), também é utilizado um modelo ARMA, sendo que as sazonalidades anuais e diárias são consideradas a partir de séries de Fourier. Já em Macedo et al. (2015), são utilizados algoritmos de mineração de dados, visando criar padrões de previsão de curva de carga que possam ser utilizados para selecionar técnicas de GLD para cada consumidor, no contexto das Smart Grids.

Outras pesquisas da literatura técnica, como as apresentadas por González-Cabrera et al. (2018) e Zhang et al. (2008), que utilizam a curva de carga em seus estudos, realizam a construção desta curva a partir da simples representação gráfica de pontos (dados da demanda diária), ou da interpolação dos mesmos, sem a definição de modelos matemáticos que descrevam a curva obtida. O mesmo é realizado em Oliveira et al. (2006), cujo estudo se dedica a investigação da relação entre os fatores de carga e perdas; para isso, as curvas diárias são transformadas em curvas de duração de carga normalizadas, sendo estas classificadas de acordo com os grupos tarifários e classes de consumo.

De modo geral, além das variações horárias e sazonais, outro aspecto importante a ser considerado na modelagem do perfil de consumo, é o tipo de carga predominante no sistema, dentre as quais destacamse as industriais e as residenciais. Nesse sentido, no cenário energético brasileiro, o setor industrial é o segmento mais influente, correspondendo a $35,7 \%$ da energia elétrica consumida (EPE; 2017). Na Fig. 1 é apresentada uma curva típica de carga (em p.u.) que descreve o consumo médio do setor industrial brasileiro. Cabe ressaltar que a demanda de potência e o comportamento da curva podem sofrer variações de acordo com a atividade industrial desenvolvida (Jardini and et al.; 2000). Observa-se que a maior demanda de potência para esta categoria ocorre nos períodos da manhã e da tarde, ou seja, em horário comercial.

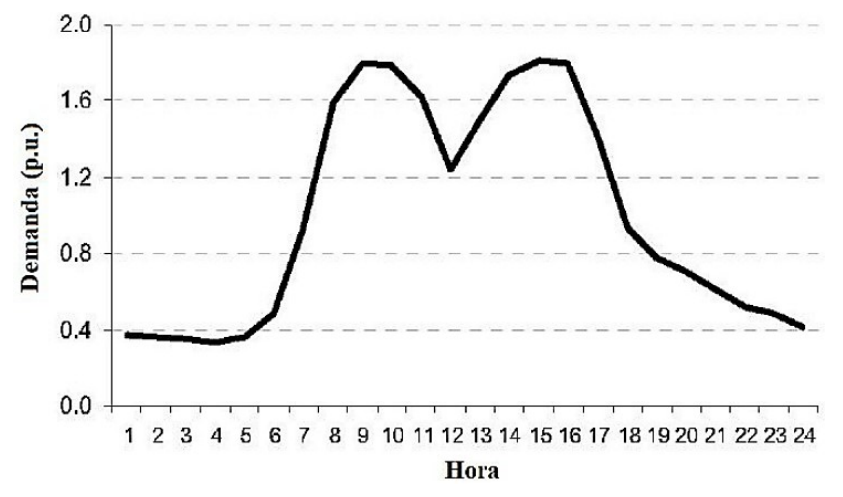

Figura 1: Curva típica de carga de consumidores industriais (Oliveira et al.; 2006)

O setor residencial, por sua vez, corresponde a 28, $8 \%$ do consumo total do Brasil (EPE; 2017). Uma curva de carga típica residencial pode ser observada na Fig. 2, onde o ponto máximo representa o horário de pico de consumo brasileiro, o qual compreende o período das 18 às 21 horas (ou das 19 às 22 horas no horário de verão). Este horário é classificado como de ponta, e compreende o período de três horas diárias consecutivas (com exceção dos finais de semana e feriados), que pode ser definido pela distribuidora, de 
acordo com a curva de carga de seu sistema elétrico (ANEEL; 2018).

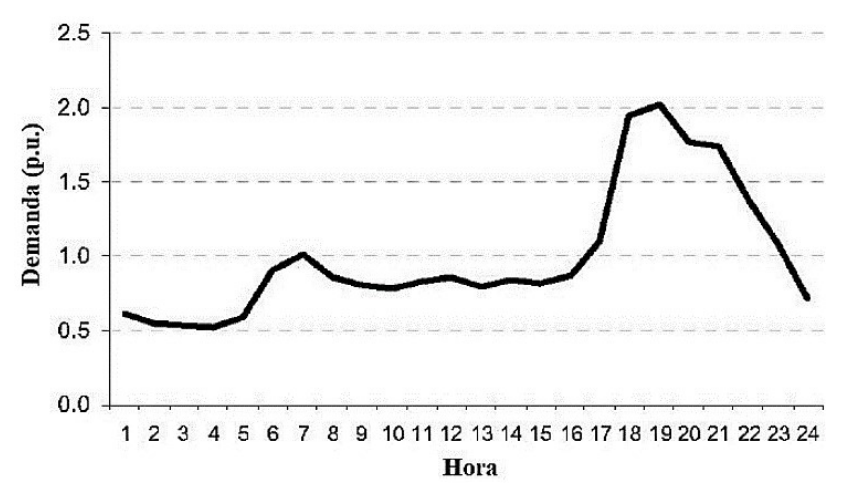

Figura 2: Curva típica de carga de consumidores residenciais (Oliveira et al.; 2006)

A demanda máxima do setor residencial está relacionada ao uso predominante, nesse horário, de equipamentos como o chuveiro e a iluminação (Hermsdorff and Filho; 2003). De modo geral, os aparelhos mais influentes na curva de carga residencial são o chuveiro elétrico e o ar-condicionado, além de aparelhos de refrigeração (Marques et al.; 2016) (Jardini and et al.; 2000). No estado do RS, devido às características climáticas, o ar-condicionado é utilizado nos extremos do inverno e verão, configurando-se como um aparelho de característica sazonal (Rahde and Kaehler; 2000). No cenário brasileiro, apesar das semelhanças, as curvas típicas de carga podem apresentar diferenças entre regiões, como o norte e o sul do país.

No horário de pico, a partir das 18 horas, a carga consumida aproxima-se da potência instalada, o que pode colocar em risco a segurança do sistema (Hermsdorff and Filho; 2003). Por outro lado, o SEP é projetado para atender esta potência máxima, mas, durante a madrugada, por exemplo, a capacidade de geração é subutilizada (ociosa), representando um investimento mal aproveitado. É justamente neste sentido que a ampliação da GD pode auxiliar no atendimento da demanda, ao mesmo tempo que reduz os custos de energia para o consumidor, e também, possibilita a redução de investimentos na ampliação da capacidade de geração centralizada e de transmissão de energia.

É neste contexto que a modelagem matemática da curva típica de carga de um sistema elétrico passa a ter significativa importância, tanto no cenário atual, para a representação, caracterização e predição do comportamento dos consumidores, possibilitando o planejamento dos sistemas, como também em um futuro próximo, quando as Smart Grids já estiverem consolidadas, e a necessidade de informações referentes ao comportamento do usuário passa a ser fundamental para o correto controle e manutenção da rede.

\section{Modelagem matemática}

Para o desenvolvimento da modelagem matemática é utilizado um conjunto de dados reais fornecidos pela concessionária de distribuição de energia elétrica. Inicialmente, é considerada a demanda dos alimentadores AL11, que possui em torno de 3000 consumidores, e AL17, com aproximadamente 4000 consumidores. Observa-se que essa concessionária possui um total de 8 alimentadores com cerca de 33000 consumidores.

A demanda desses alimentadores se refere aos anos de 2016 e 2017, sendo considerada a carga média consumida de segunda a quinta-feira, pois é possível observar, a partir dos dados, que nesses dias da semana os consumidores apresentam comportamento semelhante. Desse modo, são consideradas as variações temporais (horários do dia, dias da semana e dois anos consecutivos) e sazonais da carga, sendo que, ao abranger o período de dois anos, são consideradas duas diferentes condições climáticas para uma mesma estação, pois cada ano pode apresentar características distintas (Marques et al.; 2016).

Os dados coletados possuem uma periodicidade de aquisição de 5 minutos, e se referem a potência ativa em $k W h$, a qual foi convertida para a demanda em $k W$. A partir do cálculo da média, os dados são representados a cada 15 minutos, ao longo das 24 horas do dia, conforme indica a literatura (ANEEL; 2018) (Souza and et al.; 2010), totalizando conjuntos de 96 pontos para cada curva ajustada. Nesse sentido, a partir dos dados são calculadas as médias mensais e após, as médias por estação climática, para cada ano e alimentador.

Os modelos matemáticos e as curvas típicas são obtidas para cada estação climática (i.e., outono, inverno, primavera e verão), considerando a média dos dois anos, para cada alimentador. Para o outono, são selecionados os meses de março, abril e maio. Para o inverno, o período de junho, julho e agosto. Para a primavera o trimestre que compreende setembro, outubro e novembro. E para o verão, os meses de dezembro, janeiro e fevereiro.

Através de diagramas de dispersão das médias, é possível avaliar o comportamento dos dados. Para a realização da modelagem matemática da curva que melhor representa esses conjuntos de pontos, foram primeiramente realizados testes com funções polinomiais (considerando o grau variando de 10 até 40), gaussianas, séries de Fourier, entre outras. Dada a sazonalidade e a periodicidade dos dados, optou-se pela série de Fourier (Kreyszig; 1974), pois a partir de análises gráficas e do cálculo do coeficiente de determinação, verifica-se que um modelo composto por uma soma de $n$ funções seno, garante um melhor ajuste dos dados, com um menor número de parâmetros.

Assim, a modelagem matemática é realizada a partir de um ajuste de curvas, cuja utilização é indicada para fazer análises e extrapolações (Barroso; 1987). Considerando a importância da finalidade dessa modelagem, principalmente no que se refere ao planejamento da concessionária e dos próprios consumidores, objetivou-se atingir uma elevada acurácia, ou seja, em torno de $99 \%$. Desse modo, optou-se pela escolha de uma série de Fourier composta por oito funções seno, dada por: 


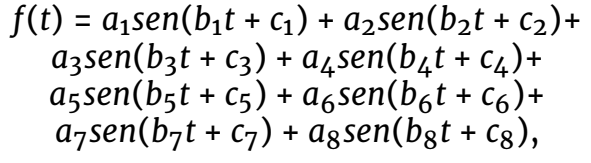

onde: $f(t)$ é a potência consumida $(k W) ; t$ é o tempo (hora do dia); $a_{1} \ldots a_{8}, b_{1} \ldots b_{8}, c_{1} \ldots c_{8}$ são os parâmetros do modelo que precisam ser estimados. Nesse caso, as curvas referentes a cada estação climática e a cada alimentador devem apresentar o coeficiente de determinação desejado. Nesse artigo, para a realização do ajuste de curvas é utilizada como ferramenta de suporte computacional o software de computação algébrica e numérica Matlab.

Desse modo, a partir dos dados reais fornecidos pela concessionária, os 24 parâmetros são obtidos através do método dos mínimos quadrados não linear, sendo aplicado o algoritmo da Região de Confiança. Para a realização do ajuste de curvas, inicialmente foi utilizado de forma uniforme e aleatória os valores iniciais padrões para os parâmetros do modelo, que estão inseridos no intervalo $(0,1)$. Além disso, também são utilizados valores padrões para o número máximo de iterações para a realização do ajuste (i.e., 400), e para as avaliações do modelo (i.e., 600). Como limite da alteração máxima em coeficientes para gradientes de diferenças finitas é estabelecido 0,1 , e para o limite da alteração mínima, $10^{-4}$. A tolerância de terminação no valor do modelo, e nos valores dos coeficientes é ajustada para $10^{-4}$.

Para avaliar o ajuste do modelo aos dados, optouse pelo cálculo do coeficiente de determinação $\left(R^{2}\right)$, visto que esta é uma medida fácil de ser obtida e que quantifica a qualidade do modelo em relação a sua capacidade de estimar corretamente os valores da variável resposta, considerando o mínimo de resíduo (erro) (Barroso; 1987). O coeficiente de determinação é dado por:

$$
R^{2}=1-\frac{S S E}{S S T},
$$

onde: $R^{2}$ é o coeficiente de determinação, SSE é a soma dos quadrados dos erros, definido por:

$$
S S E=\sum_{i=1}^{n}\left(y_{i}-\hat{y}\right)^{2}
$$

e SST é a soma dos quadrados totais, dado por:

$$
S S T=\sum_{i=1}^{n}\left(y_{i}-\bar{y}\right)^{2}
$$

onde: $y_{i}$ é a potência consumida obtida dos dados reais, $\hat{y}$ é a potência consumida a partir do modelo, $\bar{y}$ é a potência consumida média dos dados reais, e $n$ o número total de dados do conjunto.

A partir desta modelagem matemática são obtidos dois modelos matemáticos, um referente ao alimentador AL11, e outro ao AL17. Para a validação dos modelos, são considerados dois cenários distintos. Inicialmente, os dois modelos matemáticos da curva de carga média consumida nos alimentadores AL11 e AL17, são comparados com a representação gráfica dos pontos correspondentes a demanda nos mesmos alimentadores, mas considerando outro período, i.e., de outubro de 2017 a setembro de 2018. Em um segundo momento, os modelos matemáticos são comparados com o perfil de consumo médio anual de outros dois alimentadores da rede, denominados de AL12 (com perfil predominantemente industrial) e AL13 (formado por cargas residenciais e dois hospitais).

\section{Resultados e discussão}

A partir da metodologia estabelecida e da utilização do software Matlab, foram obtidos modelos matemáticos (i.e., funções) para cada estação climática e para a média geral dos dois anos, considerando cada alimentador, com diferentes coeficientes para cada parâmetro estimado. Foram realizadas várias simulações, e para todos os casos obteve-se os mesmos ajustes e coeficientes de determinação.

Na Fig. 3 são apresentados os gráficos correspondentes aos ajustes realizados para cada estação climática do alimentador AL11, considerando as médias dos anos de 2016 e 2017. Em cada gráfico, os pontos em vermelho representam a plotagem dos dados reais fornecidos pela concessionária e, a curva em azul, representa a série de Fourier ajustada para cada caso. Na Tab. 1, são apresentados os resultados das simulações, com a acurácia e o número de iterações obtidos no cálculo dos parâmetros dos modelos, o que permite verificar que o método convergiu e o modelo matemático obtido possui significativa acurácia para todas as estações.

Tabela 1: Acurácia do modelo matemático para cada estação climática referente ao alimentador AL11

\begin{tabular}{lcc}
\hline Estação climática & Acurácia $\left(R^{2} \%\right)$ & $\mathrm{N}^{0}$ de iterações \\
\hline Outono & $99,32 \%$ & 64 \\
Inverno & $98,83 \%$ & 63 \\
Primavera & $99,59 \%$ & 101 \\
Verão & $99,43 \%$ & 80 \\
\hline
\end{tabular}

Na Fig.4 são ilustradas as curvas ajustadas para as quatro estações climáticas e a média geral anual para o alimentador AL11. Quanto ao modelo matemático obtido para este alimentador (que representa a média), verificou-se que ele explica $99,58 \%$ dos dados, com um total de 77 iterações para 0 ajuste.

A partir da análise das curvas de carga da Fig. 4, é possível inferir que este alimentador é formado predominantemente por consumidores industriais, visto que apresenta maior demanda de energia elétrica no período da manhã e da tarde (em horário comercial), e que as curvas apresentam comportamento semelhante nas quatro estações climáticas, corroborando com a literatura (Oliveira et al.; 2006) (Jardini and et al.; 2000). Na Tab. 2 são apresentados os coeficientes obtidos para cada um dos parâmetros estimados para o modelo matemático que representa a curva de carga do alimentador AL11 (média), a qual é classificada como uma curva típica de carga industrial. 
(a) Outono

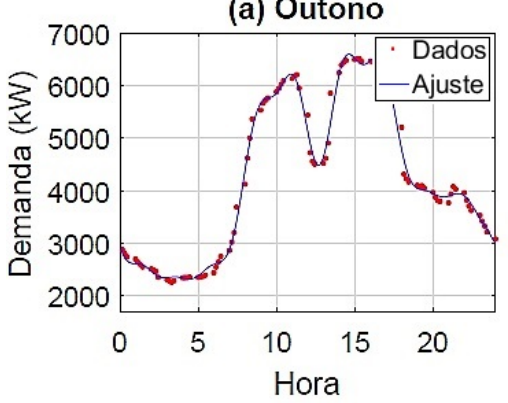

(c) Primavera

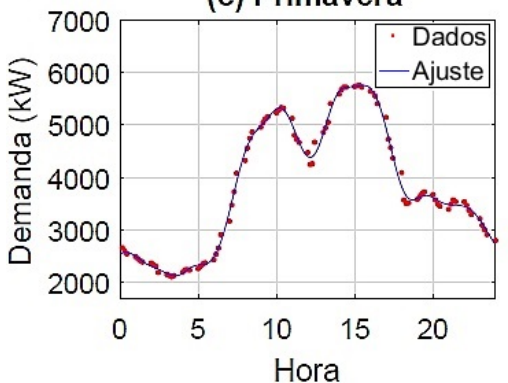

(b) Inverno

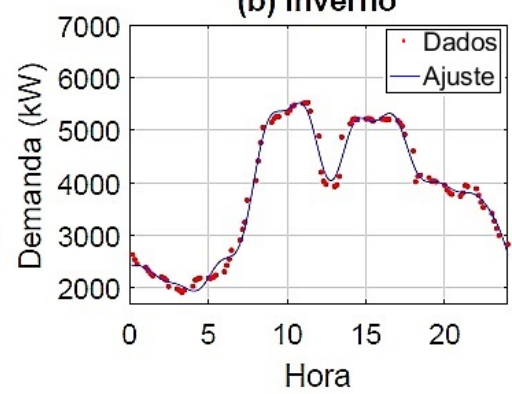

(d) Verão

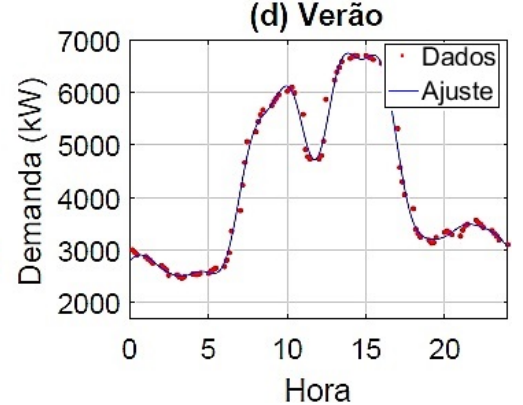

Figura 3: Ajuste da curva de carga diária de cada estação climática para o alimentador AL11

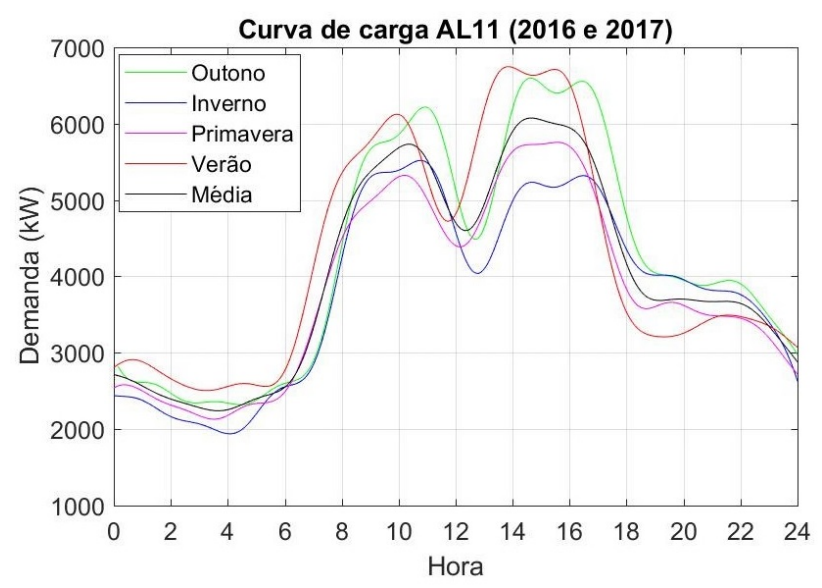

Figura 4: Curvas típicas de carga do alimentador AL11

Tabela 2: Parâmetros ajustados para o modelo da curva típica de carga industrial (AL11 - 2016 e 2017)

\begin{tabular}{cc|cc}
\hline Parâmetro & Coeficiente & Parâmetro & Coeficiente \\
\hline$a_{1}$ & 5731 & $a_{5}$ & 460 \\
$b_{1}$ & 0,1089 & $b_{5}$ & 1,089 \\
$c_{1}$ & 0,3621 & $c_{5}$ & $-2,564$ \\
$a_{2}$ & 1243 & $a_{6}$ & 50,09 \\
$b_{2}$ & 0,2 & $b_{6}$ & 1,534 \\
$c_{2}$ & 3,502 & $c_{6}$ & $-1,348$ \\
$a_{3}$ & 774,1 & $a_{7}$ & 87,16 \\
$b_{3}$ & 0,5319 & $b_{7}$ & 2,302 \\
$c_{3}$ & 1,502 & $c_{7}$ & $-5,034$ \\
$a_{4}$ & 736,4 & $a_{8}$ & 126,6 \\
$b_{4}$ & 0,7366 & $b_{8}$ & 2,003 \\
$c_{4}$ & 1,939 & $c_{8}$ & $-1,251$ \\
\hline
\end{tabular}

De modo análogo ao que foi realizado com os dados da carga do alimentador AL11, são ajustadas as curvas típicas para cada estação climática do alimentador
AL17, apresentadas na Fig. 5. Novamente verificouse, conforme apresentado na Tab. 3, que o modelo desenvolvido convergiu e apresentou significativa acurácia para todas as estações climáticas.

Tabela 3: Acurácia do modelo matemático para cada estação climática referente ao alimentador AL17

\begin{tabular}{lcc}
\hline Estação climática & Acurácia $\left(R^{2} \%\right)$ & $\mathrm{N}^{0}$ de iterações \\
\hline Outono & $99,11 \%$ & 26 \\
Inverno & $99,30 \%$ & 11 \\
Primavera & $99,58 \%$ & 31 \\
Verão & $99,36 \%$ & 172 \\
\hline
\end{tabular}

Na Fig. 6 são representadas as curvas ajustadas para as quatro estações climáticas e para a média geral anual do alimentador AL17. É possível verificar um comportamento semelhante entre as curvas, apesar das perceptíveis variações na demanda conforme o horário do dia e a estação climática. $\mathrm{O}$ modelo matemático obtido para este alimentador (considerando a média geral) representou 99, 49\% dos dados, com um total de 34 iterações.

Observando as curvas na Fig. 6, que indicam maior consumo de energia elétrica a partir das 18 horas, é possível inferir que esse alimentador é composto predominantemente por cargas residenciais (Oliveira et al.; 2006) (Jardini and et al.; 2000). O pico de consumo observado na curva, é devido, principalmente, à utilização da iluminação e do chuveiro elétrico nas residências, que ocorre de modo mais intenso entre as 18 e as 21 horas. Por outro lado, é possível verificar uma alteração desse pico no verão, ocorrendo mais tarde, entre as 19 e 22 horas (ANEEL; 2018). Nesta estação climática, também se observa uma potência consumida superior a das demais estações, e um elevado consumo de energia elétrica próximo às 15 horas, o qual se deve, possivelmente, ao uso do ar-condicionado, visto que no meio da tarde as temperaturas apresentam-se mais elevadas. 
(a) Outono

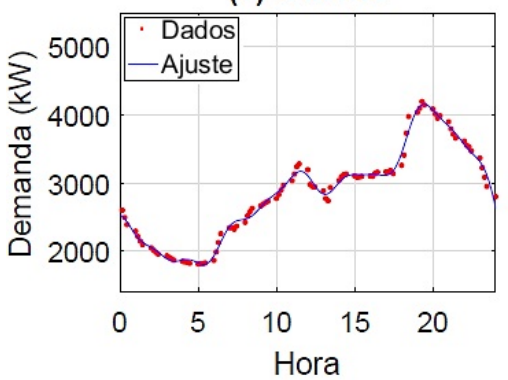

(c) Primavera

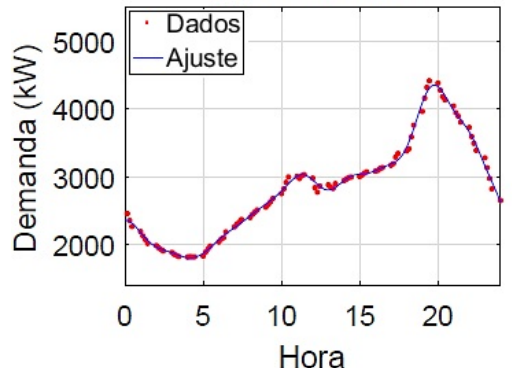

(b) Inverno

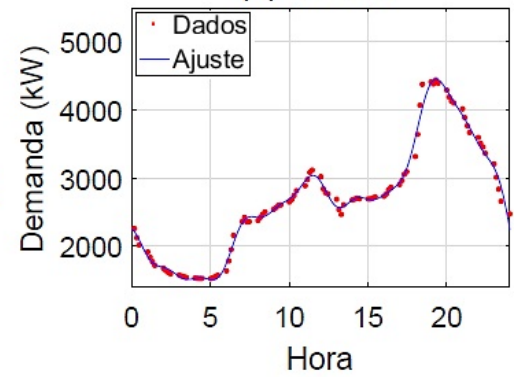

(d) Verão

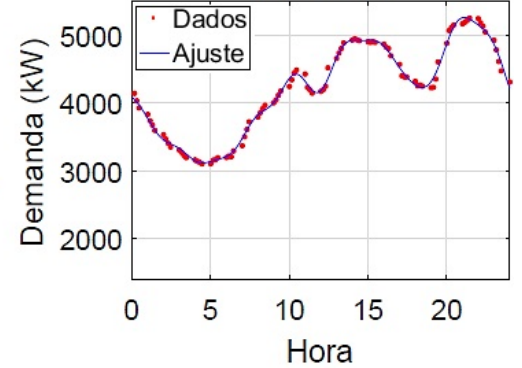

Figura 5: Ajuste da curva de carga diária de cada estação climática para o alimentador AL17

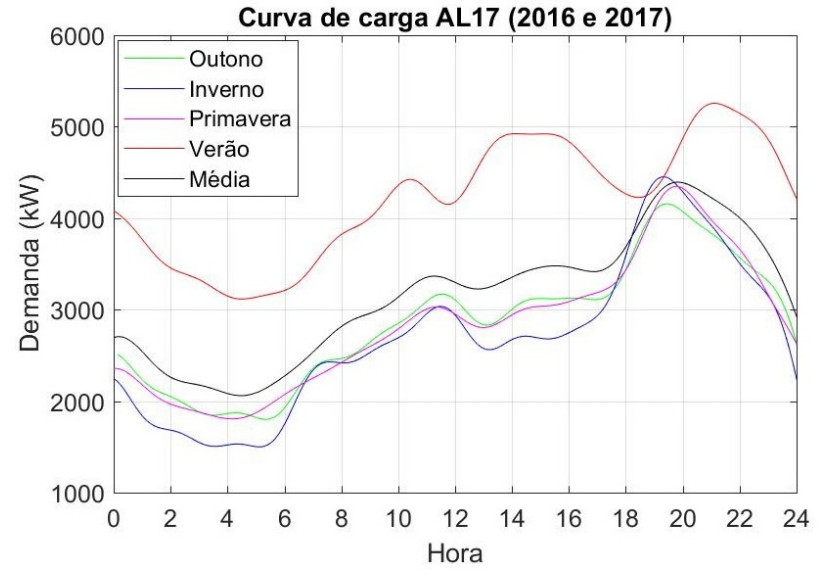

Figura 6: Curvas típicas de carga do alimentador AL17

Na Tab. 4 são apresentados os coeficientes obtidos para cada um dos parâmetros estimados para o modelo matemático que representa a curva de carga média do alimentador AL17, caracterizando-se como uma curva típica de carga residencial.

Diante do exposto, a análise dos resultados possibilita observar que os modelos matemáticos do tipo série de Fourier descrevem a demanda de potência de acordo com o horário do dia acuradamente. De modo geral, foram obtidos dois modelos matemáticos, um que descreve a curva média de carga industrial (com os coeficientes apresentados na Tab. 2 e o outro, a curva média de carga residencial (com os coeficientes apresentados na Tab. 4). Assim, objetivando a validação desses modelos na representação e na caracterização da curva típica de carga de um sistema, os mesmos são comparados considerando dois cenários de validação.

Inicialmente, os modelos que descrevem a curva média de carga para os alimentadores AL11 e
Tabela 4: Parâmetros ajustados para o modelo da curva típica de carga residencial (AL17 - 2016 e 2017)

\begin{tabular}{cc|cc}
\hline Parâmetro & Coeficiente & Parâmetro & Coeficiente \\
\hline$a_{1}$ & 5149 & $a_{5}$ & 84,78 \\
$b_{1}$ & 0,1268 & $b_{5}$ & 1,585 \\
$c_{1}$ & 0,0844 & $c_{5}$ & 2,269 \\
$a_{2}$ & 2360 & $a_{6}$ & 71,95 \\
$b_{2}$ & 0,2366 & $b_{6}$ & 1,307 \\
$c_{2}$ & 2,29 & $c_{6}$ & 1,042 \\
$a_{3}$ & 532,4 & $a_{7}$ & 24,77 \\
$b_{3}$ & 0,5099 & $b_{7}$ & 1,044 \\
$c_{3}$ & 2,578 & $c_{7}$ & 0,6865 \\
$a_{4}$ & 54,5 & $a_{8}$ & 24,67 \\
$b_{4}$ & 0,9118 & $b_{8}$ & 2,337 \\
$c_{4}$ & 0,8453 & $c_{8}$ & 0,8391 \\
\hline
\end{tabular}

AL17, foram comparados com dados reais referentes a demanda nos mesmos alimentadores, mas considerando outro período de tempo, ou seja, de outubro de 2017 a setembro de 2018. A partir da representação gráfica das curvas correspondentes aos modelos, assim como dos pontos que se referem a nova demanda em cada alimentador, percebeuse que ambas as curvas apresentavam mesmo comportamento gráfico, entretanto, a demanda de potência é distinta entre períodos e alimentadores diferentes, pois as condições climáticas podem diferir em cada ano, e os alimentadores geralmente apresentam número de consumidores desigual. Desse modo, foi desenvolvido um Fator de Correção (FC), o qual é multiplicado por todos os parâmetros $a_{n}$ (sendo $n=1,2 \ldots, 7,8)$ dos dois modelos e é definido por:

$$
F C=\frac{\bar{y}}{Y m},
$$

onde: $F C$ é o fator de correção, $\bar{y}$ é a potência consumida média diária dos dados que pretendese ajustar, e Ym é a potência consumida média 
correspondente aos modelos matemáticos (demanda média da curva diária industrial e residencial, sendo um valor constante e igual a $4037,7 \mathrm{~kW}$ e $3144,3 \mathrm{~kW}$, respectivamente). Nesse sentido, o modelo matemático (série de Fourier) que descreve a curva de carga de um sistema elétrico acuradamente, pode ser reescrito como:

$$
\begin{gathered}
f(t)=\left[a_{1} \operatorname{sen}\left(b_{1} t+c_{1}\right)+a_{2} \operatorname{sen}\left(b_{2} t+c_{2}\right)+\right. \\
a_{3} \operatorname{sen}\left(b_{3} t+c_{3}\right)+a_{4} \operatorname{sen}\left(b_{4} t+c_{4}\right)+ \\
a_{5} \operatorname{sen}\left(b_{5} t+c_{5}\right)+a_{6} \operatorname{sen}\left(b_{6} t+c_{6}\right)+ \\
\left.a_{7} \operatorname{sen}\left(b_{7} t+c_{7}\right)+a_{8} \operatorname{sen}\left(b_{8} t+c_{8}\right)\right] * F C .
\end{gathered}
$$

Os pontos correspondentes a demanda da carga nos alimentadores AL11 e AL17 para o período de outubro de 2017 a setembro de 2018, assim como as curvas de carga ajustadas para cada caso, são representados graficamente na Fig. 7. Estas curvas se referem aos modelos industrial (AL11) e residencial (AL17), e são construídas considerando os valores dos parâmetros da Tab. 2 e Tab. 4, e aplicando o FC aos parâmetros $a_{n}$. Nesse sentido, os modelos matemáticos obtidos descrevem os novos dados com $R^{2}$ de $99,45 \%$ para a curva típica industrial e de $93,84 \%$ para a curva residencial.

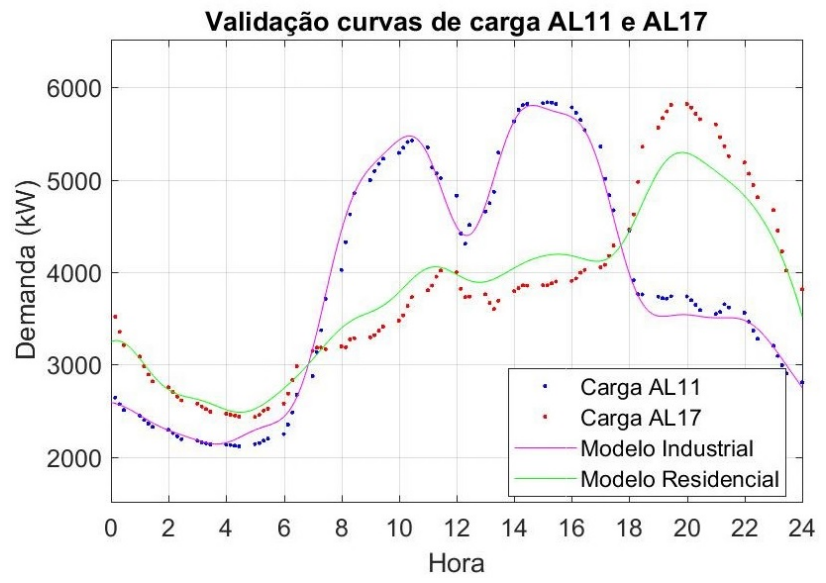

Figura 7: Validação dos modelos de curva de carga diária industrial e residencial (alimentadores AL11 e AL17) com dados do ano de 2018

O segundo cenário de validação consiste na comparação dos modelos industrial e residencial, com dados reais referentes a demanda de outros dois alimentadores da rede da concessionária, denominados de AL12 e AL13, considerando a média anual de outubro de 2017 a setembro de 2018. Na Fig. 8 são representados os pontos correspondentes à demanda nos alimentadores AL12 e AL13, e as curvas de carga obtidas a partir da equação (6), considerando os modelos industrial e residencial. Nesse caso, foram obtidos valores de $R^{2}$ de $84,59 \%$ para o ajuste entre o AL12 e a curva industrial, e de $84,69 \%$ para o ajuste dos dados do AL13 com a curva residencial. Assim, conclui-se que o alimentador AL12 é formado predominantemente por cargas industriais e o AL13 por residenciais, corroborando com as informações fornecidas previamente pela concessionária.

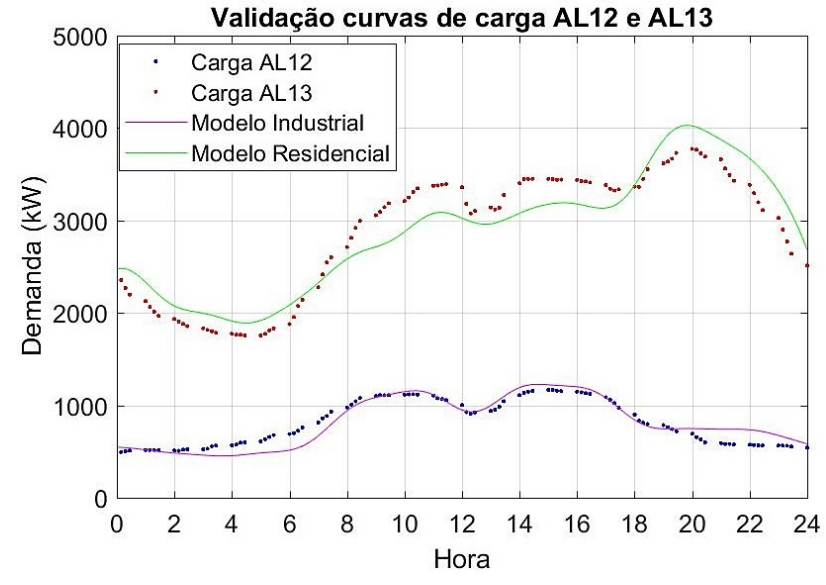

Figura 8: Validação dos modelos de curva de carga diária industrial e residencial considerando os alimentadores AL12 e AL13

Desse modo, a partir da representação gráfica da demanda média diária de um determinado sistema elétrico ou conjunto de consumidores, é possível analisar se o perfil de consumo aproximase mais de uma curva industrial ou residencial. Desse modo, pode-se definir se o sistema é formado predominantemente por cargas industriais ou residenciais. Além disso, observa-se que a curva típica de carga é descrita acuradamente pelo modelo matemático validado da equação (6), considerando os valores dos parâmetros apresentados em Tab. 2 e Tab. 4, e o FC calculado através da equação (5).

Portanto, a partir desse estudo foram obtidos dois modelos matemáticos do tipo série de Fourier, que descrevem acuradamente a curva típica de carga de um sistema elétrico real do sul do Brasil, de acordo com o tipo de carga predominante, sendo ela industrial ou residencial. Por fim, destaca-se que a modelagem matemática da curva típica de carga de um sistema e a análise da mesma, favorecem o planejamento dos sistemas de distribuição de energia elétrica, possibilitando projeções de demandas e comportamentos futuros (Balachandra and Chandru; 1999).

\section{Conclusões}

Neste artigo é realizada a modelagem matemática da curva de carga diária a partir de séries de Fourier, considerando dados reais de alimentadores de uma concessionária de energia elétrica da região sul do Brasil. A comparação das curvas típicas obtidas com as apresentadas na literatura técnica possibilitou caracterizar o alimentador AL11 como predominantemente industrial, e o alimentador AL17 como residencial. Os modelos matemáticos obtidos foram validados considerando dados referentes a demanda de potência nestes alimentadores em outro período (no ano de 2018) e também a carga consumida em outros dois alimentadores da rede elétrica da concessionária.

Desse modo, disponibiliza-se dois modelos matemáticos validados, que representam acuradamente a curva típica de carga de um sistema elétrico, permitindo a caracterização deste 
quanto ao tipo de carga predominante, sendo ela industrial ou residencial. Destaca-se ainda que a modelagem matemática da curva de carga é fundamental para as concessionárias e para os próprios consumidores, diante dos desafios da incorporação da GD e das Smart Grids, pois possibilita a obtenção de informações e favorece o gerenciamento da energia elétrica disponível.

Como possibilidades de trabalhos futuros, indica-se a aplicação dos modelos matemáticos obtidos aos demais alimentadores da rede de distribuição de energia elétrica da concessionária, assim como a possibilidade da caracterização do perfil de consumidores ou classes individuais, considerando ainda outros períodos de tempo, a partir do incremento da base de dados pela concessionária. Além disso, destaca-se que os resultados obtidos nessa pesquisa são relevantes para estudos posteriores relacionados à utilização de modelos de previsão de demanda a longo prazo, e também à modelagem matemática completa da rede de distribuição de energia elétrica.

\section{Agradecimentos}

O presente trabalho foi realizado com apoio da Coordenação de Aperfeiçoamento de Pessoal de Nível Superior - Brasil (CAPES) - Código de Financiamento 001.

\section{Referências}

Ackermann, T., Anderson, G. and Soder, L. (2001). Distributed generation: a definition, Electric Power Systems Research 57(3): 195-204. https://doi.org/ 10.1016/S0378-7796(01)00101-8.

Amjady, N. (2001). Short-term hourly load forecasting using time-series modeling with peak load estimation capability, IEEE Transactions on Power Systems 16(3). https://doi.org/10.1109/59. 932287.

ANEEL (2018). Procedimentos de Distribuição de Energia Elétrica no Sistema Elétrico Nacional - PRODIST, Brasília: Agência Nacional de Energia Elétrica. Available at http://www.aneel.gov.br/prodist (Accessed 24 May 2018).

Balachandra, P. and Chandru, V. (1999). Modelling electricity demand with representative load curves, Energy 24(3): 219-230. https://doi.org/10.1016/ S0360-5442 (98) 00096-6.

Barroso, L. e. a. (1987). Cálculo numérico (com aplicações), 2 edn, Harbra Ltda.

Deng, R., Yang, Z., Chow, M. and Chen, J. (2015). A survey on demand response in smart grids: Mathematical models and approaches, IEEE Transactions on Industrial Informatics 11(3): 570-582. https://doi.org/10.1109/TII.2015.2414719.

EPE (2017). Anuário Estatístico de Energia Elétrica 2017: ano base 2016, Empresa de Pesquisa Energética, Ministério de Minas e Energia.

Gampa, S. and Das, D. (2015). Optimum placement and sizing of dgs considering average hourly variations of load, International Journal of Electrical Power \& Energy Systems 66: 25-40. https : //doi .org/ $10.1016 / j$.ijepes . 2014.10.047.

González-Cabrera, N., Gutiérrez, G. and Gil, E. (2018). Transmission expansion planning considering an hourly demand curve, IEEE Latin America Transactions 16(3): 869-875. https://doi.org/10. 1109/TLA. 2018.8358667.

Grandjean, A., Adnot, J. and Binet, G. (2012). A review and an analysis of the residential electric load curve models, Renewable and Sustainable Energy Reviews 16(9): 6539-6565. https://doi.org/10. 1016/j.rser.2012.08.013.

Hermsdorff, W. and Filho, D. (2003). Geração independente na ponta, 3. Enc. Energ. Meio Rural .

Jardini, J. and et al. (2000). Daily load profiles for residential, commercial and industrial low voltage consumers, IEEE Transactions on Power Delivery 15(1): 375-380. https://doi.org/10.1109/ 61.847276.

Jiménez, J., Donado, K. and Quintero, C. (2017). A methodology for short-term load forecasting, IEEE Latin America Transactions 15(3): 400-407. https: //doi.org/10.1109/TLA.2017.7867168.

Kreyszig, E. (1974). Matemática Superior, Vol. 3, Livros Técnicos e Científicos.

Macedo, M., Galo, J., Almeida, L. and Lima, A. (2015). Typification of load curves for dsm in brazil for a smart grid environment, International Journal of Electrical Power \& Energy Systems 67: 216-221. https: //doi.org/10.1016/j.ijepes.2014.11.029.

Magnano, L. and Boland, J. (2007). Generation of synthetic sequences of electricity demand: Application in south australia, Energy 32(11): 22302243. https://doi.org/10.1016/j.energy. 2007.04 001.

Marques, M., Soares, F. and Russi, J. (2016). Modeling typical power demand curves using climatic data, IEEE Latin America Transactions 14(7): 3278-3284. https://doi.org/10.1109/TLA.2016.7587631.

Oliveira, M., Padilha-Feltrin, A. and Candian, F. (2006). Investigation of the relationship between load and loss factors for a brazilian electric utility, IEEE/PES Transmission \& Distribution Conference and Exposition: Latin America . https ://doi.org/10.1109/ TDCLA.2006.311390.

Rahde, S. and Kaehler, J. (2000). Modelagem da curva de carga das faixas de consumo de energia elétrica residencial a partir da aplicação de um programa de gerenciamento de energia pelo lado da demanda, ENCIT - Brazilian Congress of Thermal Sciences and Engineering .

Santos, C., Camponogara, M., Bernardon, D., Mello, A. and Pfitcher, L. (2017). Experimental platform for operational analysis of distribution networks, IEEE Latin America Transactions 15(3): 423-430. https: //doi.org/10.1109/TLA.2017.7867171. 
Souza, A. and et al. (2010). Algoritmos para estimar curvas de carga a partir de padrões de hábitos de consumo, Proceedings of the 9th Brazilian Conference on Dynamics Control and their Applications pp. 775781.

Taylor, J. (2003). Short-term electricity demand forecasting using double seasonal exponential smoothing, Journal of the Operational Research Society 54(8): 799-805. https://doi.org/10.1057/ palgrave.jors. 2601589.

Xue, Y., Chang, L. and Venkatesh, B. (2008). Energy management and control of aggregated distributed generations, IEEE - Canadian Conference on Electrical and Computer Engineering . https://doi.org/10. 1109/CCECE. 2008.4564718.

Zhang, J., Yan, A., Chen, Z. and Gao, K. (2008). Dynamic synthesis load modeling approach based on load survey and load curves analysis, IEEE - Third International Conference on Electric Utility Deregulation and Restructuring and Power Technologies pp. 10671071. https://doi . org/10.1109/DRPT. 2008.4523565. 